\title{
New Approach for Detecting and Tracking a Moving Object
}

\author{
H. Hatimi ${ }^{1}$, M. Fakir ${ }^{2}$, M. Chabi ${ }^{3}$, M. Najimi ${ }^{4}$ \\ ${ }^{1,3}$ Laboratory of Mathematics and Applications, Faculty of Sciences and Technics, University Sultan Moulay Slimane, \\ Morocco \\ ${ }^{2}$ Laboratory of Information Processing and Decision Support (TIAD), Faculty of Sciences and Technics, University \\ Sultan Moulay Slimane, Morocco \\ ${ }^{4}$ Laboratory of Biological Engineering, Faculty of Science and Technology, Sultan Moulay Slimane University, Morocco
}

\begin{tabular}{|c|c|}
\hline Article Info & ABSTRACT \\
\hline Article history: & This article presents the implementation of a tracking system for a moving \\
\hline Received Nov 12, 2017 & $\begin{array}{l}\text { target using a fixed camera. The objective of this work is the ability to detect } \\
\text { a moving object and locate their positions. In picture processing, tracking }\end{array}$ \\
\hline Revised Jan 4, 2018 & moving objects in a known or unknown environment is commonly studied. It \\
\hline Accepted Jan 20, 2018 & $\begin{array}{l}\text { is based on invariance properties of objects of interest. The invariance can } \\
\text { affect the geometry of the scene or the objects. The proposed approach is }\end{array}$ \\
\hline Keyword: & $\begin{array}{l}\text { composed of several steps; the first is the extraction of points of interest in } \\
\text { the current image. Then, these points will be tracked in the following image }\end{array}$ \\
\hline Background modeling & by using techniques for calculating the optical flow. After this step, the static \\
\hline Moving target & $\begin{array}{l}\text { points will be removed to focus on moving objects, That is to say, there is } \\
\text { only the characteristic points belonging to moving objects. Now, to detect }\end{array}$ \\
\hline Optical flow & moving targets using images of the video, the background is first extracted \\
\hline Segmentation & from the successive images. In our approach, a method of the average values \\
\hline Tracking object & $\begin{array}{l}\text { of every pixel has been developed for modeling background. The last step } \\
\text { which stays before switching to tracking moving object is the segmentation } \\
\text { which allows identifying every moving object. And by using the } \\
\text { characteristic points in the previous steps. }\end{array}$ \\
\hline
\end{tabular}

Copyright (c) 2018 Institute of Advanced Engineering and Science. All rights reserved.

\section{Corresponding Author:}

\author{
H. Hatimi, \\ Laboratory of Mathematics and Applications, \\ Faculty of Sciences and Technics, \\ University Sultan Moulay Slimane, \\ Beni Mellal, Morocco. \\ Email: h.hatimi@usms.ma
}

\section{INTRODUCTION}

Tracking a moving object is the subject of active research in computer vision [1]. Several methods are used for the detection and tracking of a moving object [2], [3], [4], using optical flow [5], [6]. Some methods are based on active contours which originate elastic models have their origins from elastic models [7], or background subtraction. Background subtraction is generally used to detect moving objects in a scene [8]. This method is to compare the current image to a static model of the scene, called the reference image, and any element present in the current image and absent in the reference image is considered mobile. There are even methods that have been proposed for the detection of moving targets by minimizing the stochastic complexity [9].

As regards the tracking of a moving object in the 2D image, several methods have been proposed. For example, there are methods which consist in describing the object to be tracked using geometric primitives as particular points [10], [11], angles, contours [12], [13], segments [14], or ellipses [15]. Active contours or snakes can also be used to determine a complex form [16]. 
To filter small periodic motions in the background, for example lighting control, Gaussian mixture model [17], and the bayesian model [18] are commonly used. In image processing and computer vision, tracking moving objects in a known or unknown environment is commonly studied. It is based on invariance properties of objects of interest. The invariance can affect the geometry of the scene or objects, the appearance of objects (that is to say photometry or color) or kinematic (such as spatial and temporal constraints).

The object of this paper is the detection and location of a deformable object in motion from a sequence of images. This research allows determining with an effective and precise method, its position in each image and determining its movement or apparent movement from one image to another. For good results, was used a camera pointed downward, its optical axis is aligned with the vertical. The rat in movement should be put in a device at the bottom of the camera in Figure 1. The problem to solve is how to collect information about the moving object during a capture sequence. This information will be the number of accesses to every zone of the open field, the mobility of rat (the time when the rat in movement) and immobility (the time when the rat stays without movement).

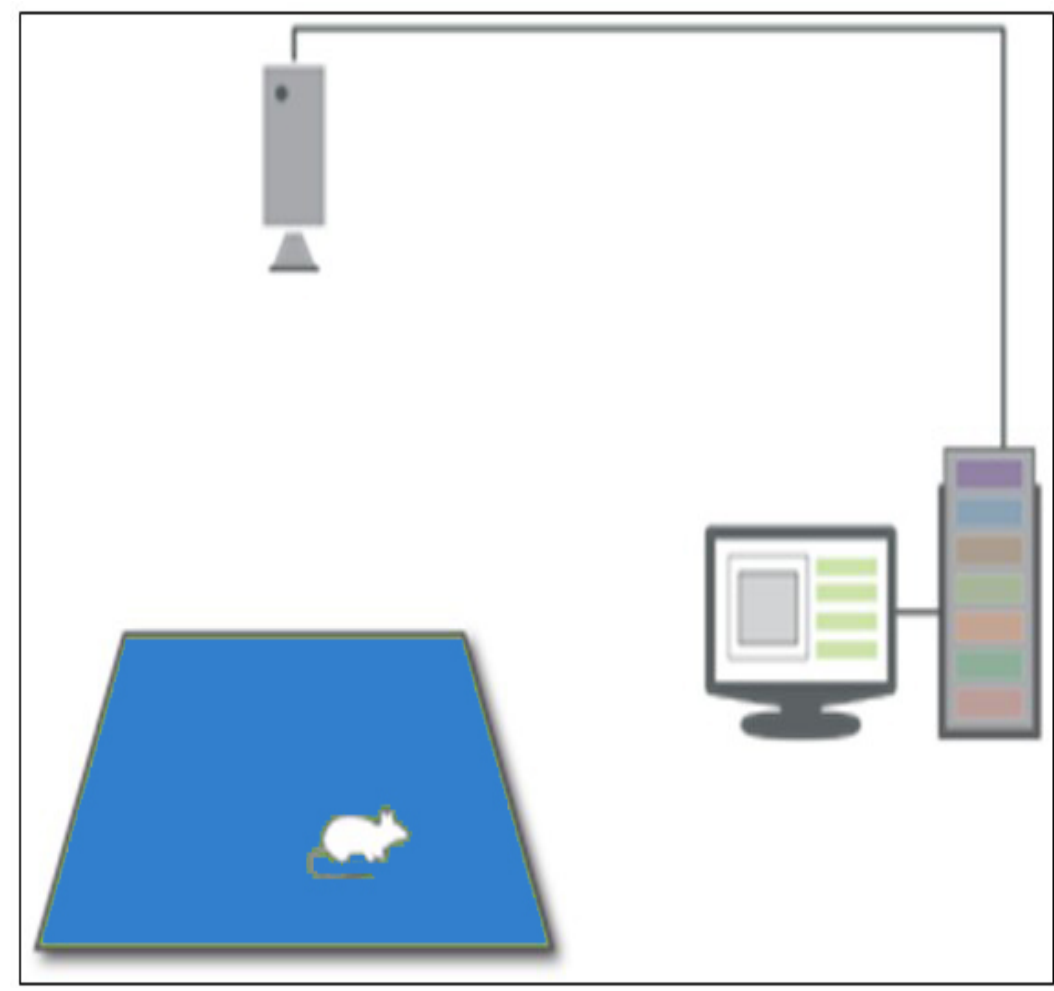

Figure 1. Motion capture systems

This paper is organized as follows: In the section II you will see the steps to follow to detect and track a moving object. In this section there is an explanation of the different steps of our approach. The first part of this section deals with extraction of interest points. The second part deals with method of calculating the optic flow. The next part deals with methods to remove immobile interest point. Next, the following section discusses the background modeling method. Finally, the last two of the section II discusses segmentation methods and Tracking the moving object. Section III represents the results of experiments conducted in the laboratory, in the end a conclusion.

\section{RESEARCH METHOD}

The step for detecting and track the moving object are illustrated in Figure 2. This approach is composed of 6 steps: 


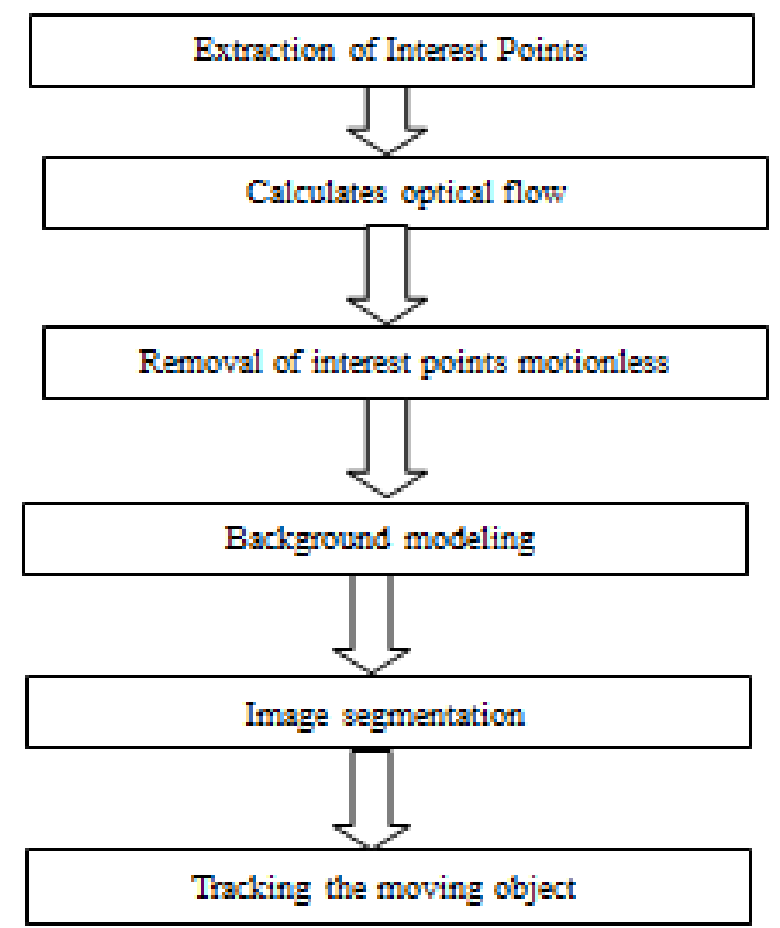

Figure 2. Effects of selecting different switching under dynamic condition

\subsection{Extracting Points of Interest}

Extraction of interest points in an image is to find the points that can be easily recognized and followed by an image to another in Figure 3. Many methods have been proposed for this simple corner detector [19] with multiscale methods, invariant in the rotations and in the changes of perspective. A number of methods have also been proposed to assess the robustness of these extractors.

For this problem, the input data of the system is always acquired by the camera. However, data output here is a list of points. It is therefore necessary to define one or more operators to build a list of points. The most commonly used for this is to extract local maxima of an image. It is also necessary to transform the input image in order to identify points of interest. We use the Harris corner detector [19] to extract the feature points which exist on the images of the video in Figure 3. Now we must eliminate the points that are motionless, items that do not belong to the moving object in the video using optical flow.

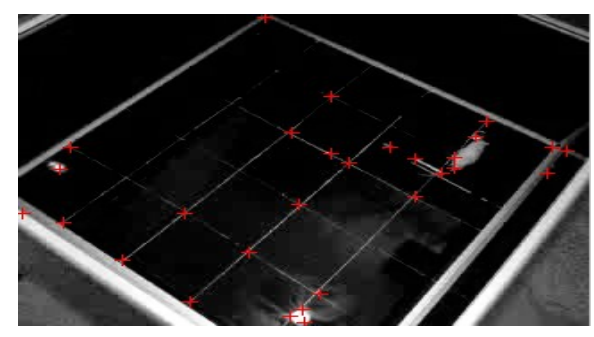

Figure 3. Extraction of interest point

\subsection{Calculation and Properties of the Optical Flow}

The motion estimation is to determine the motion vectors for predicting the processing of an image to another image in a video sequence. Motion vectors may be for the entire image or a part of the image. These motion vectors may correspond to a simple translation or generally any pattern capable of approaching the actual movement of the camera or movement of objects in the scene. 
In this work, the optical flow plays a very important role in the detection of the moving target. The purpose of this section is to introduce the concept of optical flow, properties and methods of calculation associated, and define also some properties of the optical flux used in the field of vision. This work contains taxonomy of the various methods of optical flow calculation developed since the early 80's until now. Finally, the authors determine what are the most appropriate methods and the most relevant properties to solve our problem.

The optical flow can be defined as the movement of the image on the retina (human or artificial), more precisely, it is the field of retinal velocity: the velocity of the projection space on the retina. It depends on several factors, such as the shapes of objects, their own speed, the distance or the relative speed between the object and the camera (or the eye). It is a valuable clue in human perception to among others estimate a time before collision.

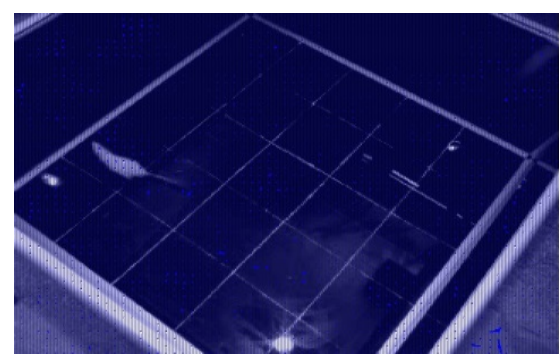

Figure 4. Extraction of the optical flow

In this section, the authors give an overview of different techniques for computing the optical flow with a detailed taxonomy. A comprehensive review of methods developed in the 80-90 years was conducted by Barron, Fleet and Beauchemin [20]. It describes in detail some of the methods presented below. The authors widely use this study to describe the oldest methods, and will complete by the most recent results. In 1981 Horn and Schunck [21], formulate what will be the basis of all differential methods: stress gradient. This constraint follows directly from the hypothesis above. Here is a quick demo: Differential translation of the hypothesis is:

$$
\frac{\partial I}{\partial t}=0
$$

That is to say

$$
\begin{aligned}
& \frac{\partial I}{\partial x} \frac{\partial x}{\partial t}+\frac{\partial I}{\partial y} \frac{\partial y}{\partial t}+\frac{\partial I}{\partial t}=0 \\
& \frac{\partial I}{\partial x} d x+\frac{\partial I}{\partial y} d y+\frac{\partial I}{\partial t}=0
\end{aligned}
$$

The equation (3) can be rewritten in another expression given in equation (4). This new equation is called "strain gradient".

$$
\vec{\nabla} I \cdot \vec{v}+\frac{\partial I}{\partial t}=0
$$

Equation (4) constrains the optical flow field $\sim \mathrm{v}$ according to the gradient of the light intensity

$$
\vec{\nabla} I=\left(\frac{\partial I}{\partial x}, \frac{\partial I}{\partial y}\right)^{T}
$$

This equation (3) gives only one constraint and therefore not possible to calculate the two components of the optical flow directly. That is why Horn and Schunck [21] impose a second constraint (called accruals) on the optical flow field. This new constraint imposes a field of more regular optical flow. The calculation of the optical flow field in this case amounts to minimize the following quantity: 


$$
\iint(\vec{\nabla} I \cdot \vec{v})+\lambda^{2}\left(\left\|\vec{\nabla} v_{x}\right\|_{2}^{2}+\left\|\vec{\nabla} v_{y}\right\|_{2}^{2}\right) d x d y
$$

In equation (6), the researchers [21] find the constraint term and a gradient according to the spatial variation of the optical flow field $\left\|\vec{\nabla} v_{x}\right\|_{2}^{2}+\left\|\vec{\nabla} v_{y}\right\|_{2}^{2}$, weighted by coefficient $\lambda$. Intuitively, minimizing the equation (6) back, first to minimize the gradient of the equation, and the spatial variation of the optical flow field. The calculation of optical flow therefore returns to the application of an optimization method minimizing this double integral.

\subsection{Tracking Points of Interest}

The major problem for tracking an object between two images is to determine the points of resemblance between the different images. The idea is to determine the feature points (corner) [19] in each image and then find the relationship between the points found in each image. These steps are used to extract a set of points of interest from an image of the video sequence using the method described in [19]. All points of interest in the image are tracked in the following image using the algorithm of Horn and Schunck [21] or optical flow [10]. The result of this operation at the instant $t$ is a set of vectors $V_{i}$ :

$$
V_{i}=\left\{X_{i}, Y_{i}\right\}
$$

Where $X_{i}$ and $Y_{i}$ are the coordinates of point $i$ relative to the reference image $I_{t}$; Now the authors must calculate the distance between the position of the point $\mathrm{i}$ in the image It and its new position in the image $\mathrm{I}_{\mathrm{t}+1}$. This step removes the static interest points that have a magnitude of movement below a minimum magnitude.

\subsection{Extraction of the Background}

To detect moving targets using panoramic images, the background scene is first extracted from successive images. The method is intended to improve the quality of the treatment compared with the basic method, but also to limit the impact of changes in the brightness of a result of extraction of background. Thus, the quality of the result is substantially enhanced. The result of the method is shown in the Figure 5.

$$
I^{\prime}(x, y)=\sum_{k=1}^{\text {nbFrame }} \frac{I_{k}(x, y)}{\text { nbFrame }}
$$

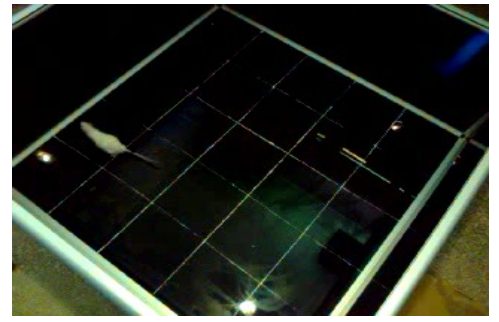

(a)

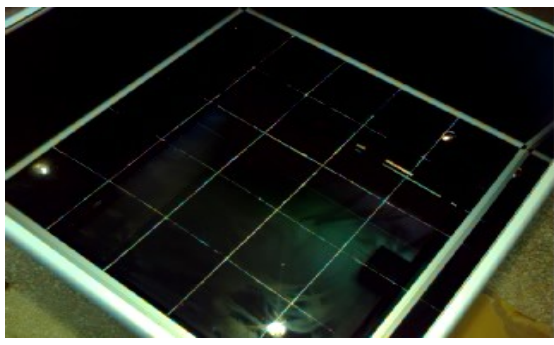

(b)

Figure 5. Extracting background; (a) Original image; (b) Background

\subsection{Segmentation}

Segmentation is one of the most studied problems in image processing [22], [23]. Its goal is the separation of the image into regions whose pixels have similar characteristics. This partitioning problem can be formulated as follows: given a cloud of $\mathrm{N}$ points in the image, the authors try to find the $\mathrm{k}$ clouds corresponding to the $\mathrm{k}$ classes of pixels. Objects after this step will classify moving object and stationary object using optical flow calculated at each point of interest. The method used in this paper constructs the regions, rather than characterizing the boundaries between regions. With this method, the authors will construct an initial state in which each pixel of the image is a unit region, and then to gradually merge related 
regions that satisfy a certain criterion of homogeneity. As an example of simple test, we can consider the difference between the mean values of pixels in the two regions. If this difference is less than a certain threshold, the authors merge the two regions into one. The result is an image in which all pixels of a region are identified by the same value. It is more natural to take for each region the average value of its pixels in the original image. We can also produce according to the coloring number of the region. More details can be found in the article [24]. The result of this step is shown in the Figure 6.

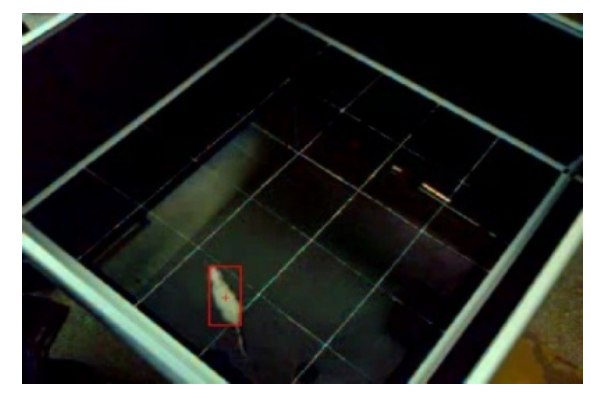

Figure 6. Detection of the target mobile

\subsection{Motion Detection}

In our work, the authors will use a reference image. The idea is to use an image of the scene devoid of objects and use them as reference image [25]. Step motion detection is important because all processes that follow, including monitoring are directly dependent on these results. In this study, the authors chose a model based pixel, which represents a good compromise between computation time /quality in Figure 7.

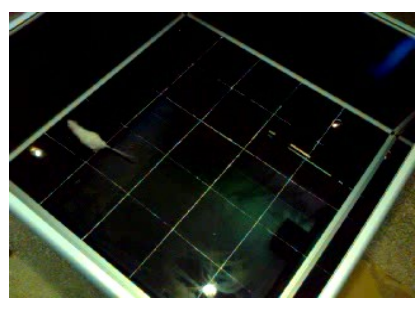

(a)

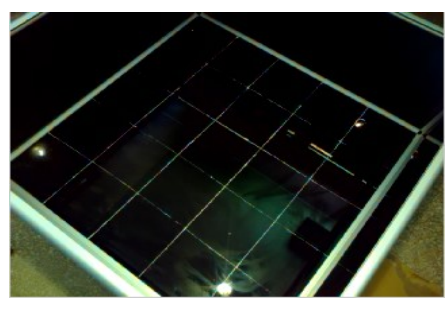

(b)

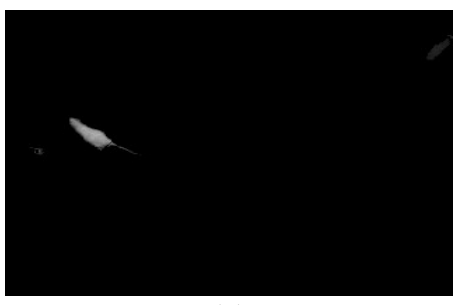

(c)

Figure 7. Difference image between the initial image and the background; (a) Initial image; (b) Background; (c) Image difference

\subsection{Object Tracking}

The tracking method will be used in this work is based on the detection of relationships between objects in the current image and the objects of the previous image, that is to say, once the objects are detected, the flow optics is calculated for each feature point belonging to the object and the center. Second, there must be correspondence between the objects in the previous image and the current image in Figure 8.

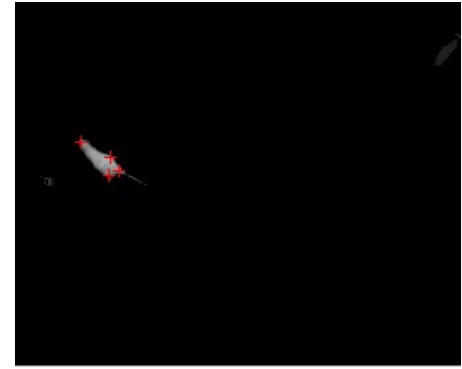

(a)

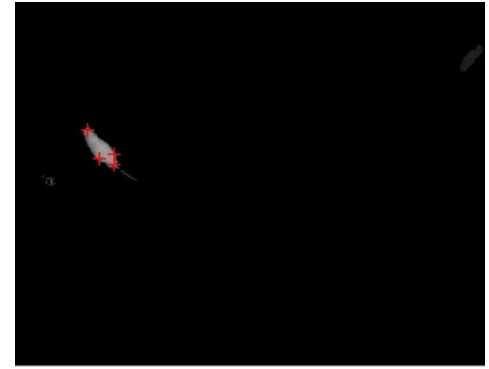

(b) 
Figure 8. Monitoring the image; (a) Image at time t; (b) Image at time t+1

\subsection{Trajectory}

Our detection system and monitoring not only allows to follow the moving object, but also to trace the path traveled by the object in Figure 9.

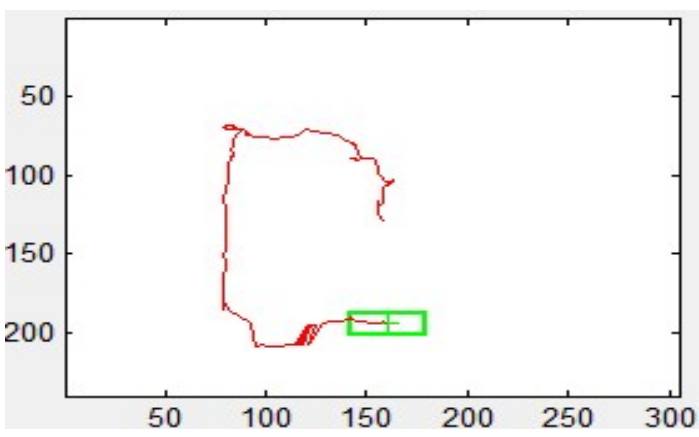

Figure 9. Drawing the trajectory

\section{RESULTS AND DISCUSSION}

To show the power of our method, we did tests with the methods using multiple features adaptive fusion [3], and method using improved detector of objects similar to target [4]. Our system has been tested on various sequences. Especially, on videos acquired in our laboratory. The authors conducted two sets of experiments indoors to assess the robustness of the algorithm to occlusions and to changes in the scene. Moreover, the scenes in which the application will be implemented mainly indoor scenes away from problems such as large lighting variations. Our interest therefore relates specifically on processes adapted to these conditions.

The proposed method in this work helped detect deformable moving objects from a video. To fully test the functionality of our program, we have made several tested with videos from 10 to 30 minutes. In all cases, our algorithm was able to locate the moving object perfectly. The detection phase gives good visual results in Figure 10, which helps a lot during the monitoring phase.

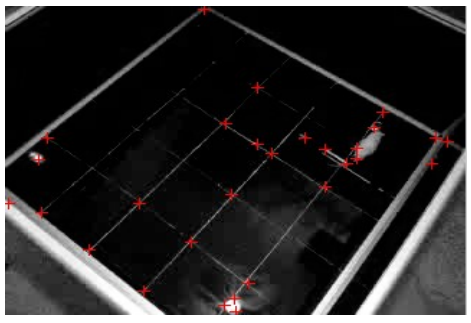

(a)

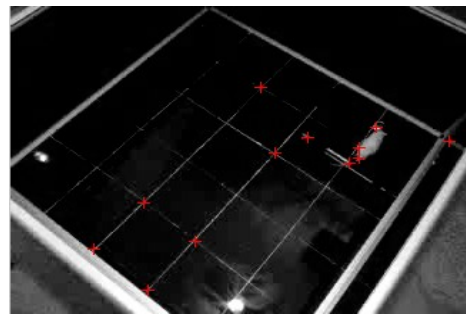

(b)

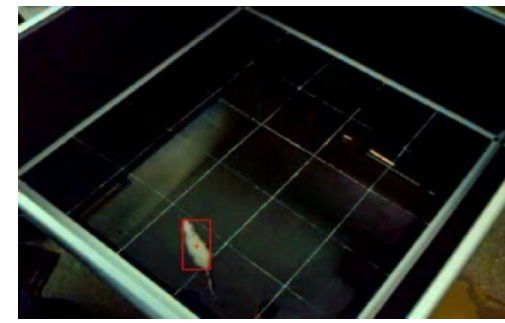

(c)

Figure 10. Process for detecting the mobile target; (a) Extracting points of interest; (b) Elimination of points of interest; (c) Segmentation and tracking of the moving object.

The final implementation of the project is to operate in minimum time as shown in Table 1, the authors looked at using simple and inexpensive methods of computation time. The program has been tested on a computer with a Core I5 processor with 4GB of ram. As we can see in table execution time is too small.

\section{CONCLUSION}

The works presented in this article handle the problem of detection and location of a mobile target. The main idea of this article is to realize of an approach for detecting automatically a moving object from a set of images, from a fixed camera. It contains a set of step to take in order to detect and track multiple moving objects. This approach can also identify the path traveled by each object, to analyze and understand 
the behavior of the object. To improve the quality of our results, a set of tests were organized to validate the application. The performances in times of calculation were assessed and given rise to some optimizations as shown in Table 1.

Table 1. Run Time of Every Operation According to the Resolution

\begin{tabular}{ccc}
\hline Operation & $640 * 480$ & $704 * 576$ \\
\hline Detection of points of interest & $0.002 \mathrm{~s}$ & 0.03 \\
Calculation of optical flow & $0.01 \mathrm{~s}$ & $0.02 \mathrm{~s}$ \\
Segmentation & $0.005 \mathrm{~s}$ & $0.007 \mathrm{~s}$ \\
Object Tracking & $0.001 \mathrm{~s}$ & $0.002 \mathrm{~s}$ \\
\hline
\end{tabular}

\section{REFERENCES}

[1] X.Zhou, W.Hu, Y.Chen, and W.Hu. Markov random field modeled level sets method for object tracking with moving cameras. Asian Conf. Compute vision. 2007; pp. 832-842.

[2] Daode Zhang, Cheng Xu, Yuanzhong Li. Robust Tracking based on Failure Recovery. Telkomnika Indonesian Journal of Electrical Engineering. 2014; Vol.12, No.2: 1005 - 1011.

[3] Jie Cao, Leilei Guo, Jinhua Wang, Di Wu. Object Tracking Based on Multiple Features Adaptive Fusion. Telkomnika Indonesian Journal of Electrical Engineering. 2014; 12(9), 6811 - 6818

[4] Shinji Fukui, Ryuji Nishiyama, Yuji Iwahori, M.K.Bhuyan, Robert J.Woodham. Object Tracking with Improved Detector of Objects Similar to Target. Procedia Computer Science. 2015; Vol 60, Pages 740-749

[5] Shoichi Araki, Takashi Matsuoaka, Naokazu Yokoya and Harua Takemura. Realtime tracking of multiple moving object contours in a moving camera image sequence. IEICE Trans. Inf. \& Syst. 2000; Vol. E83-D, No. 7.

[6] J.F. Boulanger, F. Galland, P. Martin, et P. Réfrégier. Stochastic complexity integral image based technique for fast video tracking. Optics Letters. 2008; Vol. 33 (21), pp. 2521-2523.

[7] D.J. Burr. Elastic Matching of line drawings. IEEE transaction on Pattern analysis and machine intelligence. 1981; vol. 3, n 6: pp. 708-713.

[8] Mahamuni P. D, R. P. Patil, H.S. Thakar. Moving object detection using background subtraction algorithm using simulink. International journal of research in engineering and technology. 2014; vol 03.

[9] J. Rissanen. Stochastic Complexity in Statistical Inquiry. Teaneck, NJ : World Scientific, 1989.

[10] B. Lucas, T. Kanade. An iterative image registration technique with an application to stereo vision. Proceedings of Imaging Understanding Workshop. 1981; pp. 121-130.

[11] J. Shi and C. Tomasi. Good features to track. Computer Vision and Pattern Recognition, Proceedings CVPR'94, Washington : Seattle. 1994; pp. 593-600.

[12] M.O. Berger. How to track efficiently piecewise curved contours with a view to reconstructing 3D objects. In ICPR'94. 1994; pp. 32-36.

[13] Faridah, Balza Achmad, Binar Listyana S. Lip Image Feature Extraction Utilizing Snake's Control Points for Lip Reading Applications. International Journal of Electrical and Computer Engineering (IJECE); 2015. Vol. 5, No. 4: pp. 720-728.

[14] S. Boukir, P. Bouthemy, F. Chaumette, and D. Juvin. A local method for contour matching and its parallel implementation. Machine Vision and Applications. 1998; pp. 321-330.

[15] M. Vincze. Robust tracking of ellipses at frame rate. Pattern Recognition. 2001; pp. 487 - 498.

[16] G. Hager and P. Belhumeur. Efficient region tracking with parametric models of geometry and illumination. IEEE Trans, on Pattern Analysis and Machine Intelligence. 1998; pp. 1025-1039.

[17] D. Lee. Effective Gaussian mixture learning for video background subtraction. IEEE Trans. Pattern Anal. 2005; pp. 827-832.

[18] Y. Sheikh, M. Shah. Bayesian modeling of dynamic scenes for object detection. IEEE Trans. Pattern Anal. 2005; pp. 1778-1792.

[19] C. Harris, M. Stephens. A Combined Corner and Edge Detector. Proceedings of 4th AlveyVision Conference, 1988.

[20] J. L. Barron, D. J. Fleet, and S. S. Beauchemin. Performance of optical flow techniques. International Journal of Computer Vision. 1994pp. 43-77.

[21] B.K.P. Horn et B.G. Schunk. Determining Optical Flow. Artificial Intelligence, pp. 185-203, 1981.

[22] Tahir Sag, Mehmet Çunkaş. Color image segmentation based on multiobjective artificial bee colony optimization. Applied Soft Computing. 2015; Vol 34: pp 389-401.

[23] Wen Shi, Rui-biao Zou,, Fang Wang, Le Su. A new image segmentation method based on multifractal detrended moving average analysis. Physica A: Statistical Mechanics and its Applications. 2015; Vol 432: pp. 197-205.

[24] Y.L. Tian and A. Hampapur. Robust salient motion detection with complex background for real-time video surveillance. IEEE Workshop on Motion and Video Computing. 2005; pp. 30-35.

[25] T. Yang, S.Z. Li, Q. Pan, J. Li. Real-timeand accurate segmentation of moving objects in dynamic scene. ACM Int. Workshop on video Surveillance \& Sensor Networks. 2004; pp. 136-143. 